\title{
Three-dimensional neurosonography - a novel field in fetal medicine
}

\author{
Michał Lipa ${ }^{1}$, Ritsuko Kimata Pooh ${ }^{2}$, Mirosław Wielgoś ${ }^{1}$ \\ ${ }^{1}$ Department of Obstretrics and Gynecology, Medical University of Warsaw, Poland \\ ${ }^{2}$ CRIFM Clinical Research Institute of Fetal Medicine PMC, Osaka, Japan
}

\begin{abstract}
Neurosonography is a promising technique for prenatal diagnosis, combining features of ultrasound imaging with fetal neurology. The brain is a three-dimensional structure, therefore observing brain structure in the three basic planes (sagittal, coronal and axial) is mandatory. The anterior fontanelle and sagittal suture may serve as acoustic ultrasound windows in the transvaginal brain scan, allowing to obtain high-resolution neuroimages of the intracranial structures. Furthermore, three-dimensional (3D) ultrasound combined with the transvaginal brain approach provides detailed and sophisticated neuroimages. Three orthogonal planes of the brain, tomographic ultrasound imaging (TUI) and other off-line approaches (e.g. volume contrast imaging ( $\mathrm{VCl}$ ) or HDlive silhouette imaging) may be obtained from a single 3D dataset. 3D Doppler ultrasound enables visualization of the intracerebral vascularity, allowing to obtain more precise information on cerebral perfusion. Various abnormal brain conditions, including ventriculomegaly, agenesis of the corpus callosum, posterior fossa abnormalities and others, can be well-demonstrated. Due to high rates of the associated anomalies and uncertain prognosis, any suspicion of CNS abnormalities shall imply detailed ultrasonographic evaluation of the fetal anatomy to exclude the associated anomalies. Despite a growing number of neuroimaging modalities, prenatal counselling remains a challenge as prediction of brain functionality and the neurological prognosis often remain uncertain. New investigations on the relations between various migration disorders and gene mutations, as well as recent clinical research on the relations between neuroimaging detection of local migration disorders using sophisticated imaging technologies and the postnatal neurological prognosis will contribute to the development of maternal-fetal medicine as well as pediatric neurology.
\end{abstract}

Key words: three-dimensional, ultrasound, neurosonography, fetal, brain, neurology

Ginekologia Polska 2017; 88, 4: 215-221

\section{INTRODUCTION}

Sonographic evaluation of the fetal central nervous system (CNS) has become an integral part of the prenatal screening for fetal anomalies. Rapid development of imaging techniques gave rise to neurosonography - a new field in maternal-fetal medicine. Fetal neurosonography is a promising technique for prenatal diagnosis, combining features of ultrasound imaging with fetal neurology. Owing to advanced technology of ultrasound and scanning techniques, a thorough CNS assessment can be performed, allowing for fetal abnormalities to be detected in utero. The transvaginal approach to fetal brain introduced in 1990's and the transvaginal three-dimensional (3D) ultrasound reported in 2000's have considerably contributed to the development of fetal neurosonography [1-8]. A combination of the transvaginal approach and 3D ultrasound technologies provides us with more information about fetal brain development, congenital anomalies, as well as congenital and acquired injuries. Moreover, recent advances in 3D HDlive technology and HDlive silhouette ultrasound technology have allowed for the latest neuroimaging assessment $[9,10]$.

\section{BRAIN ANATOMY ACROSS PREGNANCY}

As fetal brain undergoes constant evolution across the pregnancy, clinicians should be aware of specific brain features in each period of gestation. In the early pregnancy, three primary vesicles: prosencephalon, mesencephalon and telencephalon give rise to the future brain hemispheres, 


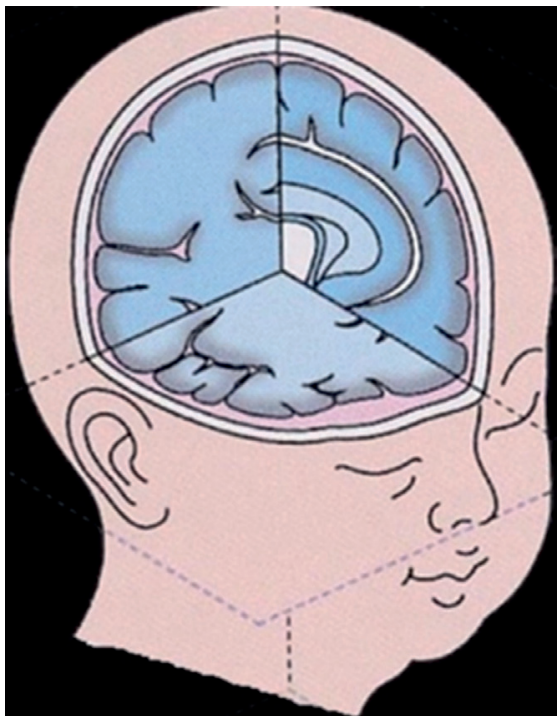

Figure 1. Three basic planes of the brain. The brain is a threedimensional structure in the sagittal, coronal and axial sections

cortex, midbrain, brainstem, cerebellum and medulla oblongata [11]. In the first trimester, the complete evaluation of CNS is not possible as many structures are not developed yet. The most important sonographic aspects of CNS evaluation across the first trimester are: choroid plexuses, midbrain and posterior fossa. On the other hand, in the second and third trimester, brain morphology may be precisely assessed with each gestational week as anatomical landmarks appear in specific periods of pregnancy. Knowledge about normal development of the fetal CNS may provide relevant and clinical data, preventing misdiagnosis of certain brain abnormalities. Owing to extensive research on the development of the fetal brain, chronological sequence of CNS development has been established. The brain is a three-dimensional structure (Fig. 1), therefore observing it in three basic planes (sagittal, coronal and axial) is mandatory.

\section{BRAIN SCREENING AND NEUROSONOGRAPHY}

Standard screening protocol for CNS malformations consists of three sectional planes: transventricular, transthalamic and transcerebellar (methodology and anatomical landmarks for each plane are described in international guidelines) [12]. Measurement of cisterna magna between 2-11 mm and atrial width of the lateral ventricles $<10 \mathrm{~mm}$ have very high negative, predictive value for the detection of brain abnormalities $[13,14]$. Using those two criteria, the majority of CNS defects may be excluded. Nonetheless, neurosonography is dedicated to advanced evaluation of the fetal brain in order to detect and differentiate brain abnormalities, especially those with resembling ultrasonographic features in routine scanning planes.

\section{TRANSVAGINAL NEUROIMAGING}

With the help of the transabdominal approach, the entire fetal CNS may be demonstrated, e.g. the brain in the axial section and the spine in the sagittal section. However, using the transabdominal approach to visualize the fetal CNS, several obstacles, e.g. maternal abdominal wall or placenta and fetal cranial bones, may render it difficult to obtain clear and detailed images.

In case of the vertex position, high-resolution transvaginal sonography remains a crucial element of advanced CNS evaluation in cases of suspected brain abnormalities. During the fetal period, immature cranial bones create wide cranial sutures and fontanelles (Fig. 2). The anterior fontanelle and sagittal suture may serve as acoustic ultrasound windows in transvaginal brain scan, enabling us to obtain high-resolution neuroimages of the intracranial structure (Fig. 3).

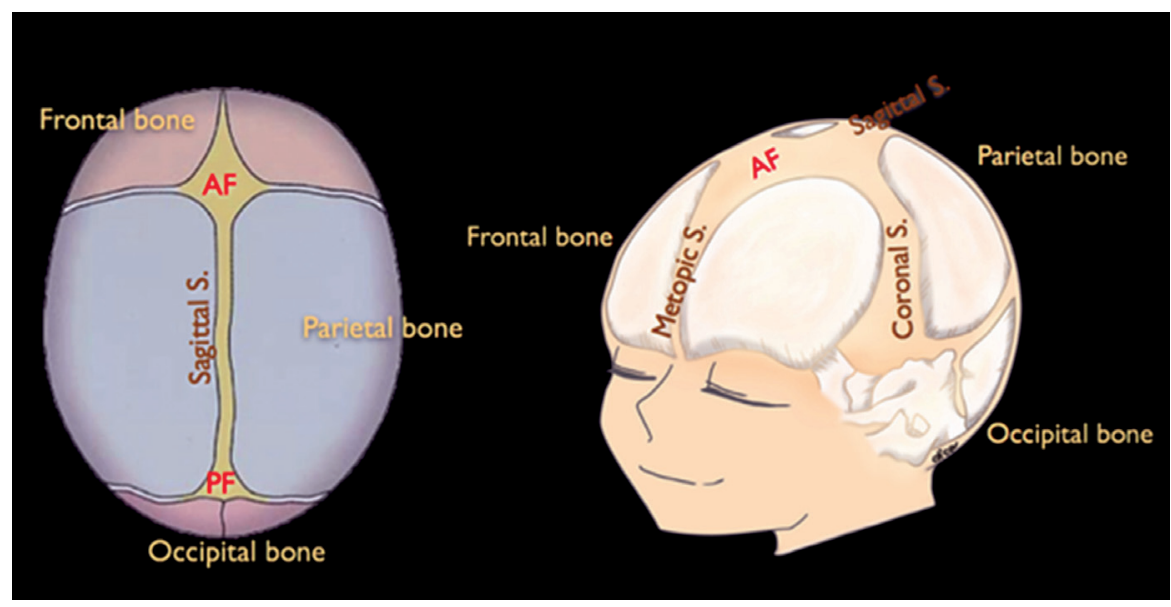

Figure 2. Cranial sutures and fontanelles. During the fetal period, immature cranial bones create wide cranial sutures and fontanelles.The anterior fontanelle and the sagittal suture can be acoustic ultrasound windows in the transvaginal brain scan; $\mathrm{AF}$ — anterior fontanelle, $\mathrm{PF}$ - posterior fontanelle) 


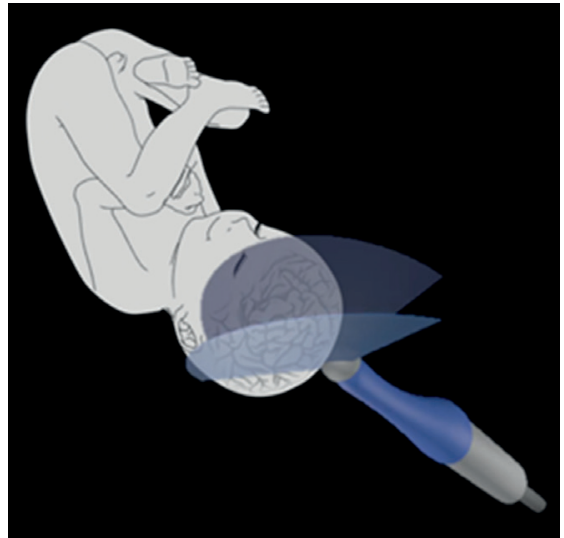

Figure 3. Transvaginal brain scan. Using the acoustic ultrasound windows of fontanelles and sagittal suture, a transvaginal brain scan provides high-resolution neuroimages of the intracranial structures

Moreover, transvaginal neurosonograms include additional scans (in coronal, para- and sagittal planes) and those images differ from the axial ones, obtained transabdominally in a routine examination.

Despite the fact that transvaginal probes provide high-resolution 2D images, the role of multidimensional modes in CNS imaging continues to grow. 3D-acquired data allow for an unlimited off-line analysis of the brain morphology. Three orthogonal planes of the brain (Fig. 4), tomographic ultrasound imaging (TUI, Fig. 5 and 6) and other off-line approaches (e.g. volume contrast imaging (VCl) or HDlive silhouette imaging) may be obtained from a single 3D dataset. 3D Doppler ultrasound enables visualization of the intracerebral vascularity (Fig. 7), allowing to obtain more precise information on cerebral perfusion. Such a wide spectrum of ultrasound neuroimaging modes raises questions about the appropriate place for fetal magnetic resonance imaging (MRI) in the diagnostic process. Several studies have been undertaken to compare detection rates

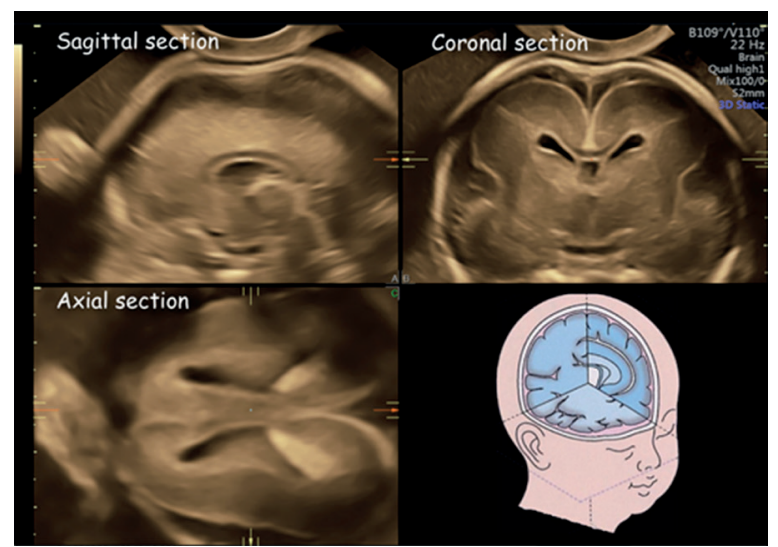

Figure 4. Three orthogonal planes of the fetal brain at 21 weeks of gestation. Sagittal, coronal and axial sections of the fetal brain appear immidiately after scanning

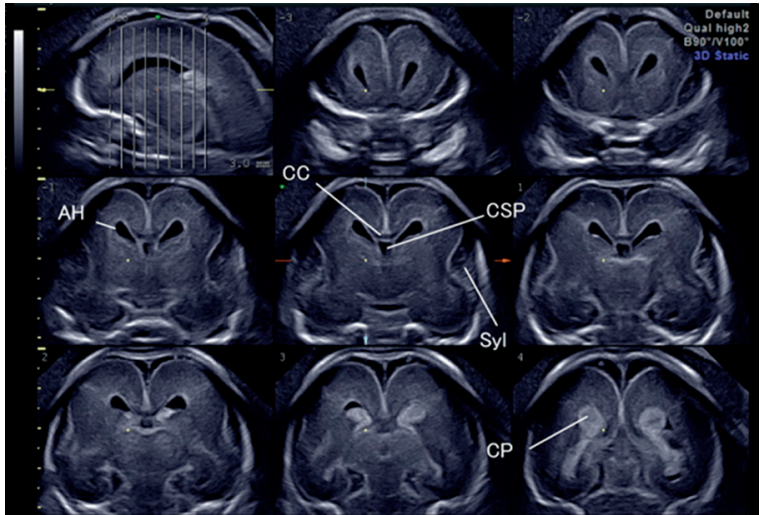

Figure 5. Tomographic ultrasound image of the coronal cutting section at 20 weeks of gestation. A series of coronal parallel cutting sections of the fetal brain is demonstrated; $\mathrm{AH}-$ anterior horn of the lateral ventricle; CC — corpus callosum; CSP — cuvum septum pellucidi; Syl —Sylvian fissure; CP — choroid plexus

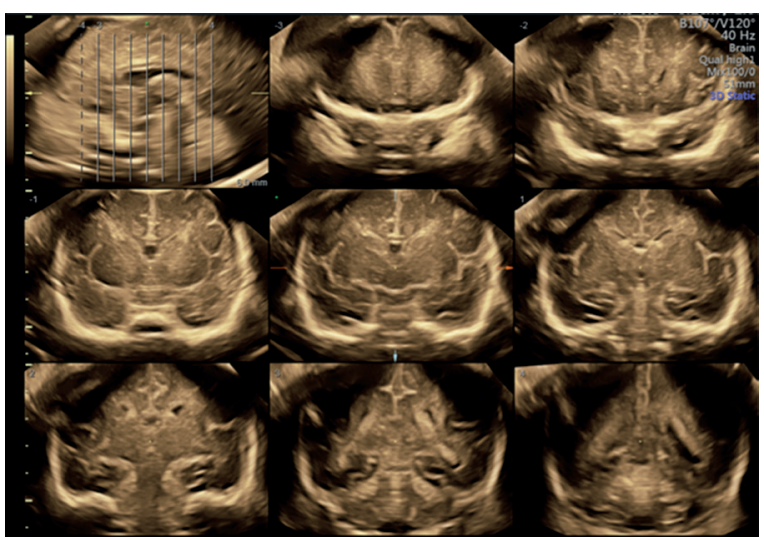

Figure 6. Tomographic ultrasound image of the coronal cutting section at 30 weeks of gestation. A series of coronal parallel cutting sections of the fetal brain is demonstrated. Note the changing appearance of the Sylvian fissure and brain surface due to cortical development, comparing to Figure 5 at 20 weeks

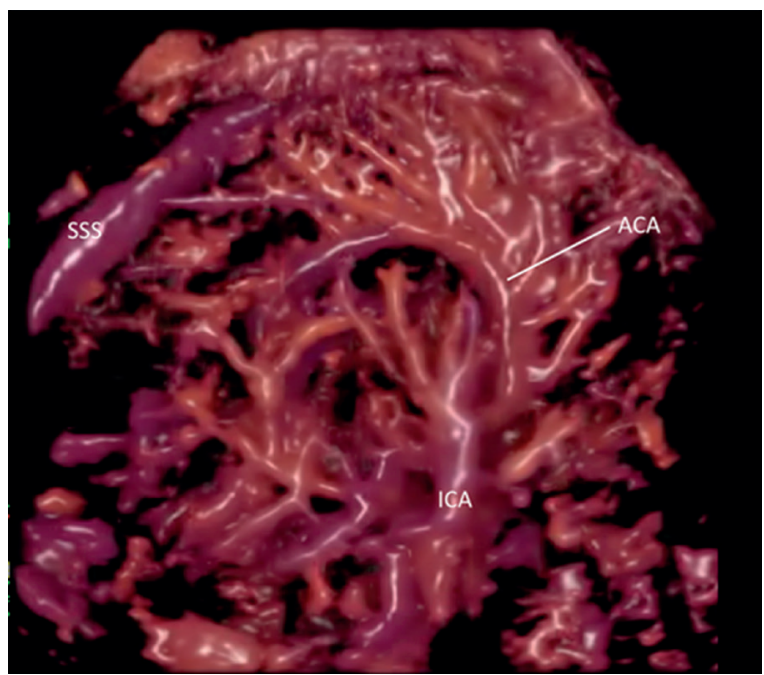

Figure 7. Normal brain vascular structure by HDlive flow imaging at 20 weeks of gestation; ACA - anterior cerebral artery, ICA -internal carotid artery, SSS - superior sagittal sinus 
of expert neurosonography (NSG) and MRI in detecting CNS abnormalities. According to the retrospective analysis by Paladini et al., the overall accuracy rates in patients who underwent expert NSG examination and MRI were $91.3 \%$ vs. $94.4 \%$, respectively. The accuracy increased when both techniques were applied (in certain malformations MRI was superior to NSG, and inversely). Both methods were concordant in $86.5 \%$ of the cases with respect to the final diagnoses. Noteworthy, NSG was more effective when performed < 24 weeks of gestation (93.1\% vs. 90.4\%) [15]. Summing up, neurosonographic multidimensional evaluation is an accurate, first-level diagnostic modality in prenatal detection of CNS malformations. In the event of an unclear diagnosis, MRI performed $>24$ weeks of gestation may provide additional data in about $5-10 \%$ of the cases $[15,16]$.

\section{CENTRAL NERVOUS SYSTEM MALFORMATIONS}

CNS malformations are one of the most common congenital defects, occurring in 1-1.6 cases per 1000 live births and even in up to $3-6 \%$ of stillbirths [17]. High incidence emphasizes the importance of screening for CNS defects in prenatal diagnosis. The most frequent CNS defects, with special emphasis on neurosonographic features, prenatal counselling and pregnancy outcomes, have been reviewed later in the article.

\section{VENTRICULOMEGALY}

Ventriculomegaly (VM) is the most common CNS finding diagnosed prenatally in fetuses with the atrial width of the lateral ventricles $>10 \mathrm{~mm}$. Neuroimages in Figures 8 and 9 present different 3D demonstrations of the fetal VM. As the etiology of VM remains variable, the examiner should investigate both, intracranial and extracranial structures in detail, perform cytogenetic/molecular genetic examination, and attempt to discover the cause of VM. Mild VM

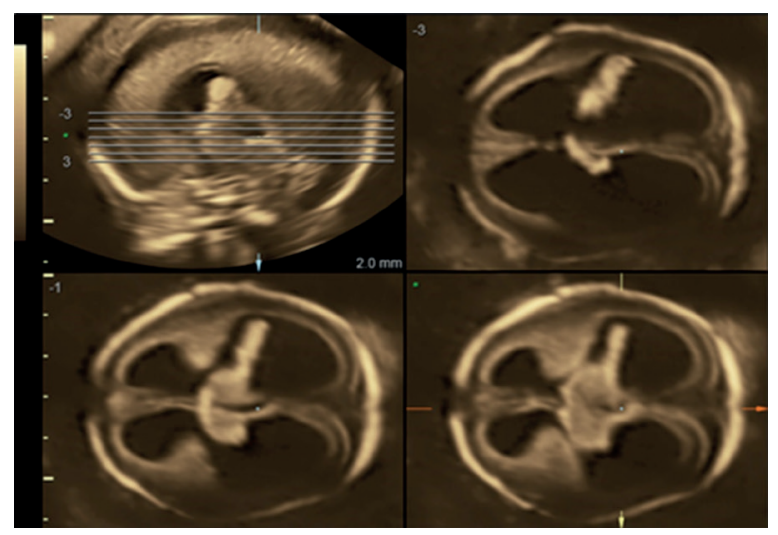

Figure 8. Tomographic ultrasound image of ventriculomegaly at 21 weeks of gestation. A series of axial cutting setions are well-depicted in a case of ventriculomegaly. Note the dangling choroid plexus depicted as hyperechogenic parts inside the enlarged ventricles

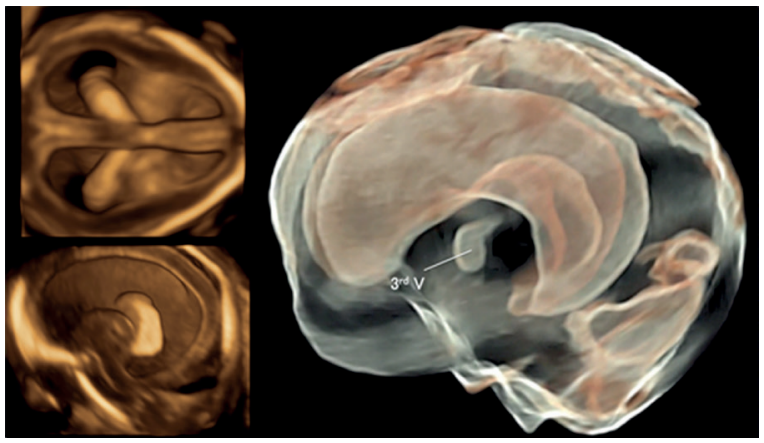

Figure 9. 3D thick slice images and silhouette image of ventriculomegaly at 16 weeks of gestation. Left upper image shows $3 D$ thick slice image in the axial cutting section and left lower image in the sagittal section. Note the mildly dangling choroid plexus (hyperechogenic parts) in the enlarged ventricles. The right image is Silhouette image. Enlarged bilateral ventricles, the $3^{\text {rd }}$ and $4^{\text {th }}$ ventricles are well-demonstrated simultaneously

(10-15 $\mathrm{mm}$ ) affects about $1 \%$ of the population, while severe VM (> $15 \mathrm{~mm}$ ) occurs in about $0.1 \%$ of the fetuses. VM is more frequent in males, however the prognosis is worse in females $[18,19]$. In clinical practice, some physicians use the terms 'ventriculomegaly' and 'hydrocephalus' alternatively for one condition - enlarged accumulation of the cerebrospinal fluid (CSF) in the ventricular system. Dilatation of the ventricles is observed in both conditions, however intracranial pressure is increased only in the hydrocephalus. Abnormal intracranial pressure and appearance of choroid plexuses may be assessed in the parasagittal and coronal views - an obliteration of the subarachnoid space and dangling choroid plexuses may not be visualized in the axial sections. Nonetheless, VM and hydrocephaly are distinct entities, although in some cases VM may progress to the hydrocephalic state [20]. Noteworthy, in many cases VM is not a symptom per se, but a symptom of brain abnormalities such as agenesis of the corpus callosum (ACC) or neuronal migration disorders. Impaired development of the brain tissue may be the reason why intracranial pressure remains normal despite enlarged ventricles [20]. Prenatal counselling in VM remains a challenge. Predictions of the pregnancy outcome and neurodevelopment in childhood may differ, depending on the severity of VM and the associated anomalies. The prognosis of isolated, mild VM remains reasonably optimistic, with a $10-15 \%$ risk of impaired neurodevelopment in adolescence. Nonetheless, coexisting CNS abnormalities are detected postnatally in $8-40 \%$ of the cases. Unfavorable prognostic factors include female sex, atrial width of $>12 \mathrm{~mm}$, asymmetry of ventricular dilatation (> $2 \mathrm{~mm}$ ), progressive VM (15\% of the cases), and associated abnormalities [19]. In fetuses with VM and other abnormalities, especially with genetic syndromes, the rate of an adverse pregnancy outcome is significantly higher and the prognosis is poor. 

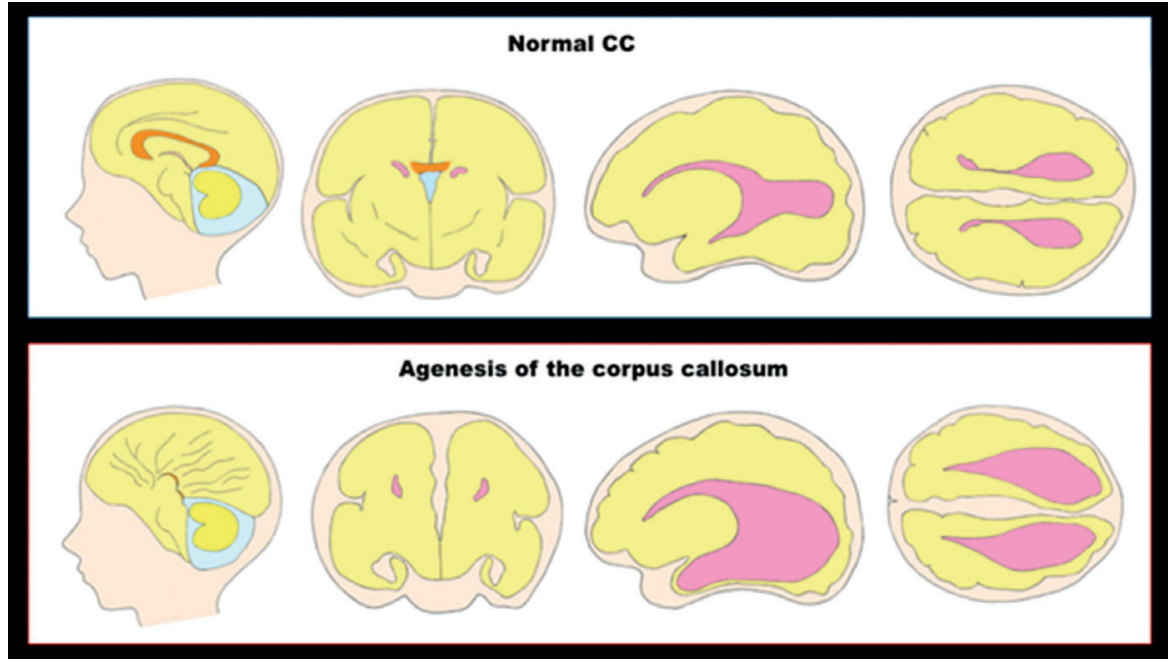

Figure 10. Schematic pictures showing four major features of agenesis of the corpus callosum. Midsagittal, anterior coronal, parasagittal and axial views from the left. Upper pictures are normal structures for comparison

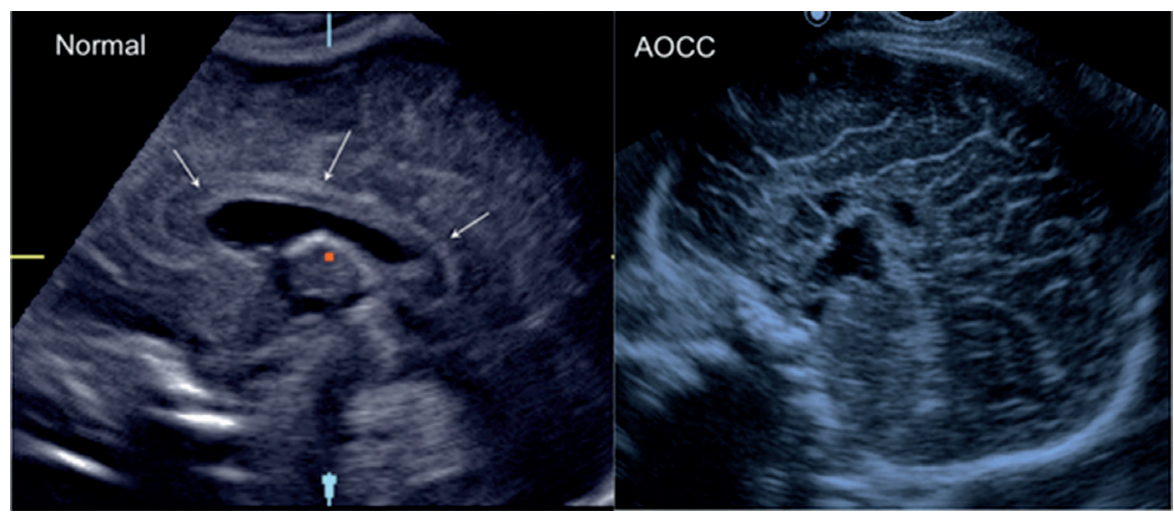

Figure 11. Mid-sagittal image of normal and agenesis of the corpus callosum. Left; normal corpus callosum (arrows) is well-demonstrated. Right; agenesis of the corpus callosum (ACC). Note the absence of the corpus callosum and cingulate gyrus and sulci in the radial pattern instead

\section{AGENESIS OF CORPUS CALLOSUM (ACC)}

The corpus callosum (CC) is the main forebrain commissure, transmitting motor, sensorial and cognitive information between the left and the right cerebral hemispheres. Its development starts from gestational week 7. By the end of week 20, CC may be visualized on ultrasound. At this stage, the shape resembles CC in adults, however the functional development will not be completed until the second year of postnatal life [21]. Prenatal diagnosis of hypogenesis or agenesis of CC presents a considerable challenge, as sonographic markers are not specific or may be not present during a routine second-trimester examination. Absent cavum septum pellucidum, colpocephalic ventriculomegaly (tear-shaped posterior horns of the lateral ventricles) and parallel appearance of the anterior horns may indicate ACC. The major features of ACC have been shown in Figure 10. In the sagittal (Fig. 11) and coronal (Fig. 12)

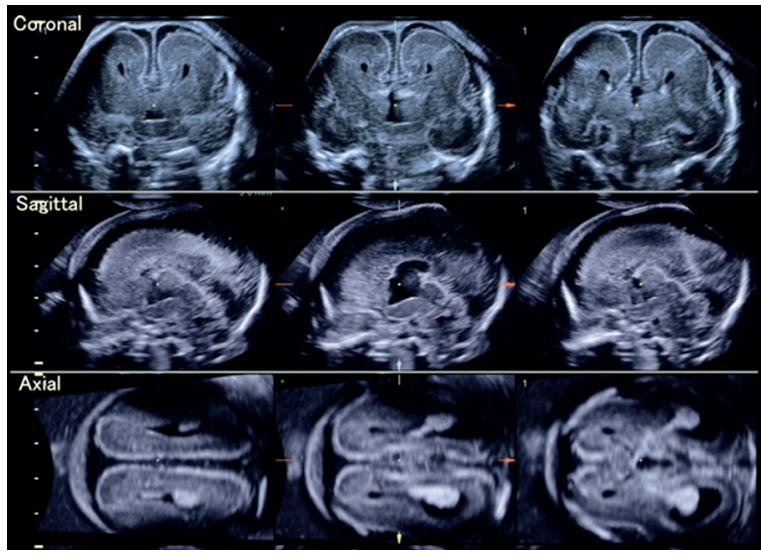

Figure 12. Tomographic ultrasound images in a case of agenesis of the corpus callosum at 20 weeks of gestation. Coronal, sagittal and axial parallel cutting sections are demonstrated from the above. Note the absence of the corpus callosum in the coronal and sagittal sections and parallel anterior horns in the axial section 
planes, it is relatively easy to make an accurate diagnosis of agenesis or hypogenesis of CC, but it is difficult in routine screening in the axial plane. Paladini et al., in the retrospective analysis of fetuses with CC defects, revealed that in most cases the abovementioned ultrasound signs were not present $<24$ weeks of gestation. On the other hand, they were more evident with the advancing gestational age: VM was present in $74.3 \%$ and colpocephaly in $68.6 \%$ of the cases in the late second trimester [22]. Prediction of the long-term pregnancy outcome and neurodevelopment in childhood depends on the type of defect and presence of the associated anomalies. In about $50 \%$ of the cases, ACC is an isolated finding and in the other half, the associated anomalies (both, cerebral and extra-cerebral) are found [23]. Due to the low frequency of prenatally diagnosed ACC in the general population and low rate of prenatal detection, the results, methodology and follow-up periods in studies on pregnancy outcome and neurodevelopment are heterogenic. Mangione et al., in their meta-analysis, revealed normal development in $71 \%$ of the newborns with isolated ACC [23]. On the other hand, the prognosis for fetuses with ACC and other anomalies remains poor. To sum up, prenatal detection of CC malformations presents a challenge due to the fact that sonographic signs may be very subtle or appear after the second-trimester scan [22]. Proper management requires thorough neurosonographic evaluation of CNS. When ACC is suspected both, cerebral and extra-cerebral abnormalities should be excluded and karyotyping ought to be offered. MRI plays an important role in the diagnostic process and is necessary to confirm the primary diagnosis.

\section{POSTERIOR FOSSA ABNORMALITIES}

The cerebellum is an important, midline structure of the posterior fossa, responsible for linguistic, perceptive and motor functions. Embryological development starts in the early first trimester and continues up to the end of the first year of postnatal life. Noteworthy, the theory published by Robinson et al., regarding the development of the posterior fossa, may shed some light on the current state of knowledge and approach to certain abnormalities. These authors suggest that there might be a continuum in posterior fossa anomalies involving the fourth ventricle and the Blake's pouch [14].

The group of the most frequent lesions, commonly referred to as the'Dandy-Walker continuum or spectrum', comprises of Dandy-Walker malformation (DWM), megacisterna magna (MCM), and the Blake's pouch cyst (BPC). As distinct defects associated with various pregnancy outcomes may have similar ultrasonographic appearance, prenatal differentiation remains an issue for the sonographers [24]. Despite challenging diagnosis, the current classification of the posterior fossa defects is even more confusing - the

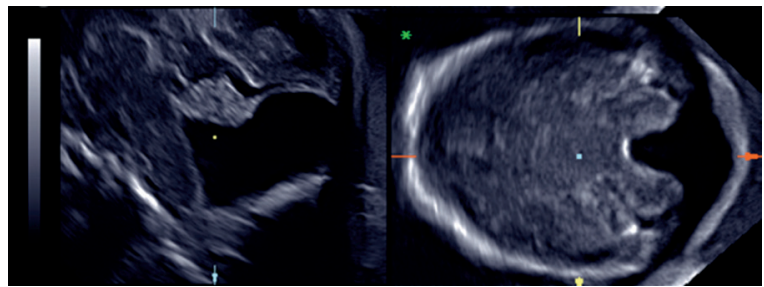

Figure 13. Sagittal (left) and axial (right) images of the Dandy Walker Malformation from 3D orthogonal views at 26 weeks of gestation. Note the typical features of DWM: cystic dilatation of the Blake's pouch, vermian hypoplasia and the upward movement of the tentorium

terminology and the knowledge about this group of CNS defects continue to evolve $[25,26]$. Nonetheless, some studies report up to $90 \%$ accuracy of neurosonography, when performed by experts [24].

DWM is a very rare congenital malformation, with the incidence of 1 to 25.000-35.000 [27]. Typical features of DWM include cystic dilatation of the Blake's pouch, vermian hypoplasia, and upward movement of the tentorium (Fig. 13). Ventriculomegaly is a frequent finding in fetuses with DWM. Sagittal scans, preferentially in multiplanar modes, remain to be the key elements of prenatal differential diagnosis. Volpe et al., reported that the measurements of brainstem-vermis (BV) and brainstem-tentorium (BT) angles provide crucial data to categorize cases with upward rotation of the vermis in the second trimester. According to the results, values of the BV angle of $>45$ degrees strongly suggest DWM, whereas measurements of $<30$ degrees indicate BPC [28]. Posterior fossa structures comprise of higher number of neurons as compared to the rest of the cerebellum. This fact makes the prediction of the pregnancy outcome and postnatal development quite difficult [27]. The findings of the studies on pregnancy outcomes in DWM and vermian hypoplasia/agenesis are inconsistent due to heterogeneous and confusing terminology. Various authors used to classify specific entities in different ways. Unfortunately, a reliable and comprehensive summary of the results is not possible. In general, the prognosis for fetuses with DWM, other CNS and extra- CNS abnormalities remains poor [24]. The outcome is more favorable in isolated defects with normal vermis lobulation [27]. Some individuals may remain asymptomatic [29]. Nonetheless, even in isolated DWM, many neurological complications may occur, including epilepsy, hypotony or mental retardation [30].

\section{CONCLUSIONS}

Detailed neurosonograms provide valuable data about the morphology of the fetal brain. Systematic, high-resolution transvaginal approach for the evaluation of fetal CNS with the use of multidimensional imaging techniques may significantly increase the overall detection rate of CNS 
defects. Due to high rates of the associated anomalies and uncertain prognosis, any suspicion of CNS abnormalities shall imply detailed ultrasonographic evaluation of the fetal anatomy to exclude associated anomalies. Despite the steadily growing number of neuroimaging modalities, prenatal counselling remains a great challenge as the prediction of brain functionality and the neurological prognosis are often uncertain. Further studies on the relations between migration disorders and gene mutations, together with the recent clinical research on the relations between neuroimaging detection of local migration disorders using sophisticated imaging technologies and postnatal neurological prognoses, will contribute to the development of maternal fetal medicine as well as pediatric neurology.

\section{REFERENCES}

1. Timor-Tritsch IE, Monteagudo A. Transvaginal fetal neurosonography: standardization of the planes and sections by anatomic landmarks. Ultrasound Obstet Gynecol. 1996; 8(1):42-47, doi: 10.1046/j.1469-0705.1996.08010042.x, indexed in Pubmed: 8843619.

2. Monteagudo A, Reuss ML, Timor-Tritsch IE. Imaging the fetal brain in the second and third trimesters using transvaginal sonography. Obstet Gynecol. 1991; 77(1): 27-32, indexed in Pubmed: 1984223.

3. Monteagudo A, Timor-Tritsch IE, Moomjy M. In utero detection of ventriculomegaly during the second and third trimesters by transvaginal sonography. Ultrasound Obstet Gynecol. 1994; 4(3): 193-198, doi: 10.1046/j.1469-0705.1994.04030193.x indexed in Pubmed: 12797179.

4. Pooh RK, Nakagawa Y, Nagamachi N, et al. Transvaginal sonography of the fetal brain: detection of abnormal morphology and circulation. Croat Med J. 1998; 39(2): 147-157, indexed in Pubmed: 9575270.

5. Pooh RK. Three-dimensional ultrasound of the fetal brain. In Kurjak A. [eds] Clinical application of 3D ultrasonography. Parthenon Publishing, Carnforth. 2000: 176-180.

6. Pooh RK, Pooh K, Nakagawa Y, et al. Clinical application of three-dimensional ultrasound in fetal brain assessment. Croat Med J. 2000; 41(3): 245-251, indexed in Pubmed: 10962041.

7. Timor-Tritsch IE, Monteagudo A, Mayberry P.Three-dimensional ultrasound evaluation of the fetal brain: the three horn view. Ultrasound Obstet Gynecol. 2000; 16(4): 302-306, doi: 10.1046/j.1469-0705.2000.00177.x, indexed in Pubmed: 11169304.

8. Monteagudo A, Timor-Tritsch IE, Mayberry P.Three-dimensional transvaginal neurosonography of the fetal brain:,navigating ' in the volume scan. Ultrasound Obstet Gynecol. 2000; 16(4): 307-313, doi: 10.1046/j.1469-0705.2000.00264.x, indexed in Pubmed: 11169305.

9. Pooh RK. Brand new technology of HDlive silhouette and HDlive flow images. In: Pooh RK, Kurjak A. ed. Donald School Atlas of Advanced Ultrasound in Obstetrics and Gynecology. Jaypee Brothers Medical Publishers Private Limited, New Delhi 2015: 1-39.

10. Pooh R. Recent advances in 3D ultrasound, silhouette ultrasound, and sonoangiogram in fetal neurology. Donald School Journal of Ultrasound in Obstetrics and Gynecology. 2016; 10: 193-200, doi: 10.5005/jp-journals-10009-1468.

11. Chaoui R, Nicolaides KH. Detecting open spina bifida at the 11-13-week scan by assessing intracranial translucency and the posterior brain region: mid-sagittal or axial plane? Ultrasound Obstet Gynecol. 2011; 38(6):609-612, doi: 10.1002/uog.10128, indexed in Pubmed: 22411445.

12. International Society of Ultrasound in Obstetrics \& Gynecology Education Committee. Sonographic examination of the fetal central nervous system: guidelines for performing the,basic examination' and the,fetal neurosonogram! Ultrasound Obstet Gynecol. 2007; 29(1): 109-116, doi: 10.1002/uog.3909, indexed in Pubmed: 17200992.

13. Filly RA, Cardoza JD, Goldstein RB, et al. Detection of fetal central nervous system anomalies: a practical level of effort for a routine sonogram. Radiology. 1989; 172(2): 403-408, doi: 10.1148/radiology.172.2.2664864, indexed in Pubmed: 2664864.

14. Robinson AJ. Inferior vermian hypoplasia--preconception, misconception. Ultrasound Obstet Gynecol. 2014; 43(2): 123-136, doi: 10.1002/uog.13296, indexed in Pubmed: 24497418.

15. Paladini D, Quarantelli M, Sglavo G, et al. Accuracy of neurosonography and MRI in clinical management of fetuses referred with central nervous system abnormalities. Ultrasound Obstet Gynecol. 2014; 44(2): 188-196, doi: 10.1002/uog.13243, indexed in Pubmed: 24186262.

16. Malinger $G$, Lev $D$, Lerman-Sagie T. Is fetal magnetic resonance imaging superior to neurosonography for detection of brain anomalies? Ultrasound Obstet Gynecol. 2002; 20(4): 317-321, doi: 10.1046/j.1469-0705.2002.00825.x, indexed in Pubmed: 12383310.

17. Onkar D, Onkar P, Mitra K. Evaluation of Fetal Central Nervous System Anomalies by Ultrasound and Its Anatomical Co-relation. J Clin Diagn Res. 2014; 8(6): AC05-AC07, doi: 10.7860/JCDR/2014/8052.4437, indexed in Pubmed: 25120962.

18. Pilu G, Falco P, Gabrielli $S$, et al. The clinical significance of fetal isolated cerebral borderline ventriculomegaly: report of 31 cases and review of the literature. Ultrasound Obstet Gynecol. 1999; 14(5): 320-326, doi: 10.1046/j.1469-0705.1999.14050320.x, indexed in Pubmed: 10623991.

19. Ouahba J, Luton $D$, Vuillard $E$, et al. Prenatal isolated mild ventriculomegaly: outcome in 167 cases. BJOG. 2006; 113(9): 1072-1079, doi: 10.1111/j.1471-0528.2006.01050.x, indexed in Pubmed: 16956339.

20. Pooh RK. Ventriculomegaly. In: Pooh RK, Kurjak A. ed. Fetal neurology. Jaypee Brothers Medical Publishers, New Delhi 2009: 62-74.

21. Vasudevan C, McKechnie L, Levene M. Long-term outcome of antenatally diagnosed agenesis of corpus callosum and cerebellar malformations. Semin Fetal Neonatal Med. 2012; 17(5): 295-300, doi: 10.1016/j. siny.2012.07.001, indexed in Pubmed: 22840681.

22. Paladini D, Pastore G, Cavallaro A, et al. Agenesis of the fetal corpus callosum: sonographic signs change with advancing gestational age. UItrasound Obstet Gynecol. 2013; 42(6):687-690, doi: 10.1002/uog.12506, indexed in Pubmed: 23671008.

23. Mangione R, Fries N, Godard P, et al. Neurodevelopmental outcome following prenatal diagnosis of an isolated anomaly of the corpus callosum. Ultrasound Obstet Gynecol. 2011;37(3): 290-295, doi: 10.1002/uog.8882, indexed in Pubmed: 21337654.

24. Gandolfi Colleoni G, Contro E, Carletti A, et al. Prenatal diagnosis and outcome of fetal posterior fossa fluid collections. Ultrasound Obstet Gynecol. 2012; 39(6): 625-631, doi: 10.1002/uog.11071, indexed in Pubmed: 22173885.

25. Parisi MA, Dobyns WB. Human malformations of the midbrain and hindbrain: review and proposed classification scheme. Mol Genet Metab. 2003; 80(1-2): 36-53, indexed in Pubmed: 14567956.

26. Malinger G, Lev D, Lerman-Sagie T. The fetal cerebellum. Pitfalls in diagnosis and management. Prenat Diagn. 2009; 29(4): 372-380, doi: 10.1002/pd.2196, indexed in Pubmed: 19194867.

27. Klein O, Pierre-Kahn A, Boddaert N, et al. Dandy-Walker malformation: prenatal diagnosis and prognosis. Childs Nerv Syst. 2003; 19(7-8): 484-489, doi: 10.1007/s00381-003-0782-5, indexed in Pubmed: 12879343.

28. Volpe P, Contro E, De Musso F, et al. Brainstem-vermis and brainstem-tentorium angles allow accurate categorization of fetal upward rotation of cerebellar vermis. Ultrasound Obstet Gynecol. 2012; 39(6): 632-635, doi: 10.1002/uog.11101, indexed in Pubmed: 22253138.

29. Bolduc ME, Limperopoulos C. Neurodevelopmental outcomes in children with cerebellar malformations: a systematic review. Dev Med Child Neurol. 2009; 51(4): 256-267, doi: 10.1111/j.1469-8749.2008.03224.x, indexed in Pubmed: 19191827.

30. Garel C, Moutard ML. Main congenital cerebral anomalies: how prenatal imaging aids counseling. Fetal Diagn Ther. 2014; 35(4): 229-239, doi: 10.1159/000358519, indexed in Pubmed: 24577226. 